\title{
Viability of different tumor cell lines after treatment with complexes of oligoribonucleotides with D-mannitol
}

\section{Ivanna Kraievska, Zenoviy Tkachuk}

\section{Institute of Molecular Biology and Genetics NAS of Ukraine}

\section{Introduction}

Complexes of oligoribonucleotides with D-mannitol (ORNs-D-m) have a wide range of biological activity: antiviral, antioxidant, immunomodulatory, and others (Melnichuk et.al., 2018; Marchyshak et.al., 2018). They are obtained by the formation of complexes between the D-mannitol and total yeast RNA. It was found that the drug contains oligoribonucleotides of different lengths; the predominant fraction is from 2 to 15 nucleotides. Previous studies on the murine model of B16 melanoma have shown that solid tumor formation was not observed with the simultaneous administration of melanoma cells and ORNs-D-m (Kraievska et.al., 2019).

The aim of this work was to study the effect of ORNs-D-m on the viability of different tumor cell lines in vitro.

\section{Materials and methods}

Murine B16 melanoma and human U251 glioblastoma were used for studies. Cells $\left(8-10 \times 10^{3}\right.$ cells in $100 \mu \mathrm{L}$ medium per well) were plated in 96-well plates and treat with different substances. At the end of various treatments, MTT solution was added to each well, incubated and samples were read by an ELx800 Absorbance Microplate Reader at a wavelength of $570 \mathrm{~nm}$. Cell viability was determined relative to intact control, taken as $100 \%$ viability (Fig.1). For representation on graphs, concentration data were logtransformed and the percent viability data in time-dependent graphs were fit to a nonlinear regression curve (log (inhibitor) vs response). A comparison of the effect of compounds was carried out by the concentration of half-maximal inhibition of $\mathrm{IC}_{50}$, which was determined based on dose-dependent curves. Results are expressed as the mean \pm standard deviation. The statistical significance between the groups was determined by an unpaired t-test. $\mathrm{P}<0.05$ was considered to indicate a statistically significant difference. All analyses were performed using the GraphPad Prism 8.0.1 software.

Treatment with:

ORNs-D-m

- ORNS Acid forms

- ORNs sodium salt

ORNs-D-m sodium salt - Salt forms

D-mannitol

dissolution in culture medium

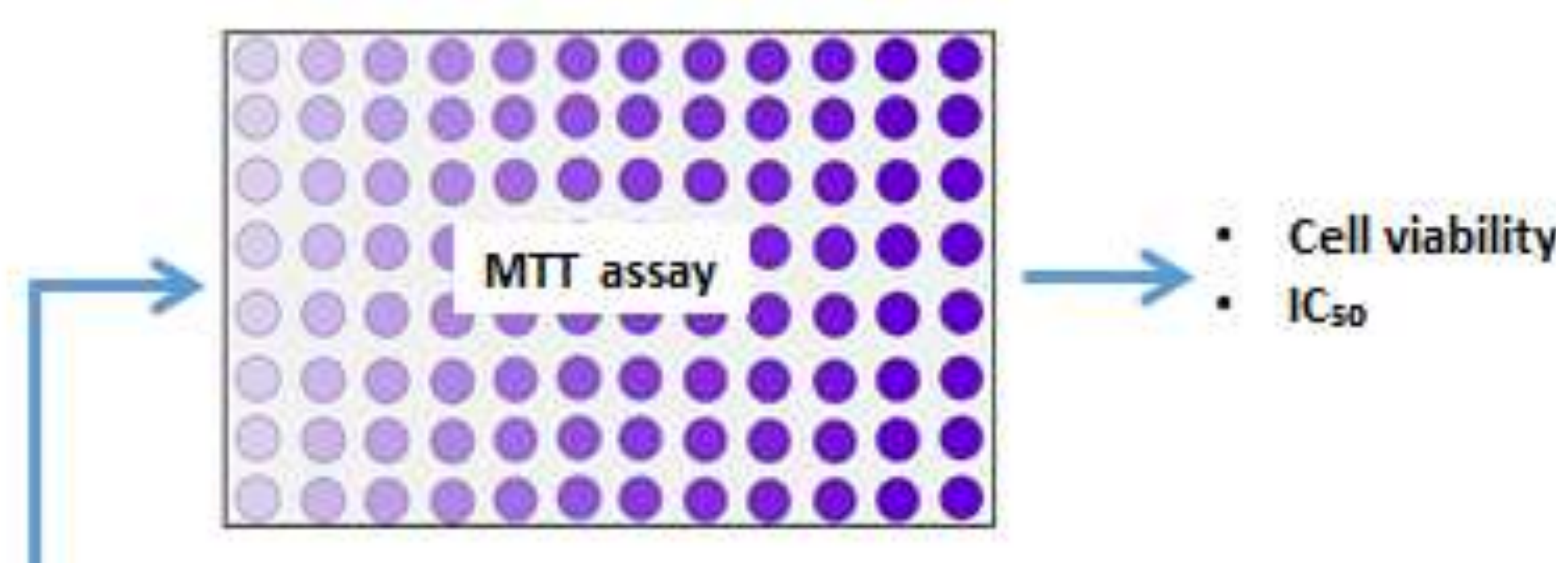

\section{Results}

- Murine B16 melanoma

The duration and dose of ORNs-D-m treatment effects on B16 viability. Thus, the viability of cells was significantly reduced after 48-hours treatment compared to 12-hours treatment, with $\mathrm{IC}_{50}$ $2.7 \pm 0.2 \mathrm{mg} / \mathrm{ml}$. In contrast, the $\mathrm{IC}_{50}$ with ORNs treatment was 2,5 times higher, which indicates their less pronounced effect compared to ORNs-D-m. It was found, that the acid form of these substances is crucial for the inhibitory effect on cancer cells because sodium salts of ORNs-D-m and ORNs have no inhibitory influence on cell growth (Fig.2).
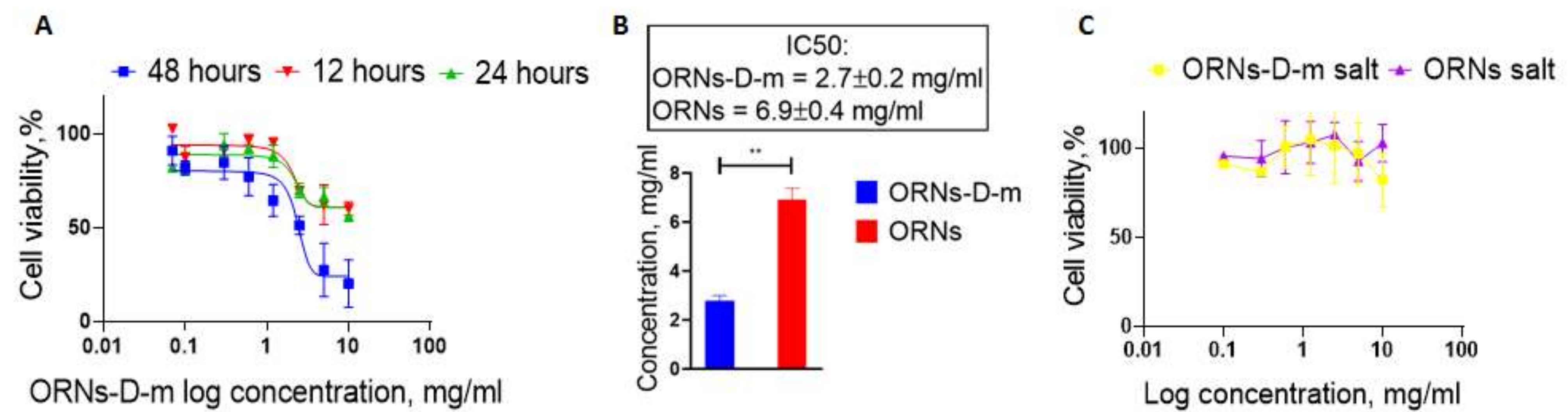

Fig.2. (A) Concentration- and time-dependent viability after treatment with ORNs-D-m

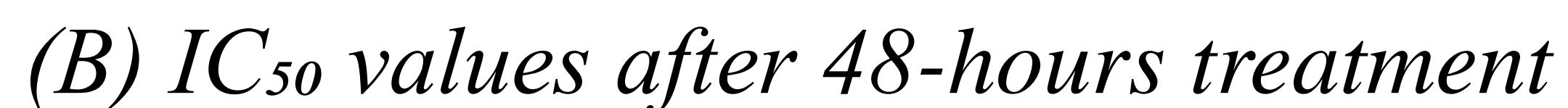

(C) No growth inhibition after 48 hours treatment with salt forms

\section{- Human U251 glioblastoma}

For the U251 cell line, a similar trend in viability after treatment was found, with $\mathrm{IC}_{50}$ for ORNs-D-m $-1.2 \pm 0.04 \mathrm{mg} / \mathrm{ml}$, and for ORNs - in 8 times higher. As well, the level of cell viability after 48-hours treatment with salt forms of these substances for applied concentrations was higher, than $70 \%$, which indicates the absence of an inhibitory effect (Fig.3).

D-mannitol in concentration $2,8 \mathrm{mg} / \mathrm{ml}$ (amount in the highest dose of ORNs-D-m) did not affect both cell lines viability.
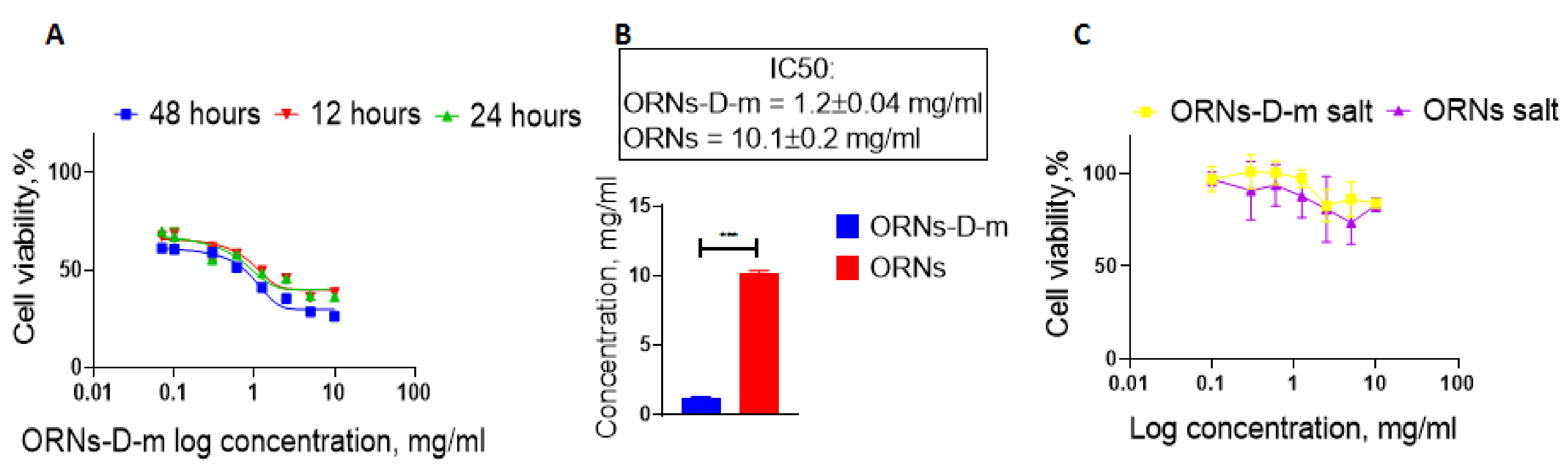

Fig.3. (A) Concentration- and time-dependent viability after treatment with ORNs-D-m

(B) IC $C_{50}$ values after 48-hours treatment

(C) No growth inhibition after 48 hours treatment with salt forms

\section{Conclusions}

- ORNs-D-m inhibits the viability of both cancer cell lines: murine B16 melanoma and human U251 glioblastoma, in dose- and timedependent manner.

- ORNs show a less inhibitory effect on viability.

- Acid forms demonstrate an inhibitory effect on the viability of cancer cells in contrast to salt forms, which don't show this influence in applied concentrations. 